\title{
Perceived anxiety and plasma cortisol concentrations following rock climbing with differing safety rope protocols
}

\author{
C I Hodgson, N Draper, T McMorris, G Jones, S Fryer, I Coleman
}

University of Chichester, Chichester, UK

\section{Correspondence to:} Christopher I Hodgson, University of Chichester, College Lane, Chichester, P019 6PE, UK; c.hodgson@chi.ac.uk

Accepted 22 January 2008 Published Online First 14 April 2008

\begin{abstract}
Objectives: To examine how different safety rope protocols impact on subjective anxiety and self-confidence levels and plasma cortisol concentrations and the relationship between subjective states and cortisol during rock climbing.
\end{abstract}

Methods: Participants $(n=12)$ were tested in three climbing conditions that were designed to invoke low, moderate and high physical and mental stress. Plasma cortisol concentrations were collected before and after climbing and participants reported subjective anxiety and self-confidence states for each climb.

Results: Repeated measures analysis of variance showed significant differences between conditions for somatic anxiety $\left(F_{2,22}=7.74, p=0.009\right)$, self-confidence $\left(F_{2}\right.$. $\left.{ }_{22}=9.52, p=0.001\right)$ and change in plasma cortisol concentration $\left(F_{2,22}=3.71, \mathrm{p}=0.041\right)$. Preplanned polynomial comparisons showed that these were linear effects; somatic anxiety was higher in the higher stress conditions whilst self-confidence was lower. Plasma cortisol concentration change was also linear. Regression analyses showed cubic relationships between plasma cortisol concentration and cognitive anxiety $\left(R^{2}=0.452\right)$, self-confidence $\left(R^{2}=0.281\right)$ and somatic anxiety $\left(R^{2}=0.268\right)$.

Conclusions: There is a relationship between plasma cortisol concentration and subjective anxiety and selfconfidence states during rock climbing. Changes in the way the safety rope is organised can impact on anxiety, cortisol concentration and self-confidence during rock climbing.

Rock climbing is a recreational adventure sport with physical and psychological demands. Climbers ascend a defined route which means using a limited number of holds and features on the wall for support and progression. This creates a physical demand on the performer but difficult climbing also places significant demands on cognitive processing and emotional control. Cognitive processing tasks include technical decisions about how to complete the moves between holds and tactical decisions including speed of climbing, the use of resting and recovery positions, and when to clip the safety rope into anchors. Technical and tactical decisions can be compromised if a climber exhibits poor emotional control, resulting in errors including clipping the rope from inefficient positions, missing opportunities to rest, and making moves in a less than optimal fashion.

Most studies on the demands of rock climbing have concentrated on the physical elements of the task and physical preparation for climbing. ${ }^{1}$
Previous research ${ }^{2}{ }^{3}$ has explored lactate accumulation and clearance strategies but there is no published research on subjective anxiety and physiological stress responses to climbing and no study has examined how different climbing protocols might impact on stress and emotions. Williams, Taggart, and Carruthers ${ }^{4}$ measured adrenaline and noradrenaline concentrations during rock climbing and found significant increases in adrenaline but not noradrenaline following a single climb on a sandstone outcrop. No attempt was made to relate this to emotional experience or the specific task demands of climbing.

Typically studies that have examined the demands of rock climbing tasks ignore any psychological element and treat climbing as a purely physical task. Clearly some physical responses such as heart rate and endocrine activity are likely to be a result of a combination of physical and psychological factors. Different climbing protocols can have a large impact on the physical, cognitive and emotional demands that a climber experiences even whilst climbing identical routes. During lead climbing a climber must complete the moves between holds and manage their own safety rope by clipping it into anchors on route. This style of climbing is considered the best way to ascend a rock climbing route $e^{5}$ and is the ideal to which climbers aspire. A climber failing to complete their route will generally fall a short distance until the slack rope in the system is taken up and they are caught. This can mean falling up to 3-4 m depending on venue and position on the route. A common training protocol called top roping involves climbing a route with a safety rope threaded through an anchor at the top of the route. During this style of ascent the climber does not need to attend to the safety rope so can concentrate on the physical moves in a secure setting. Failing to complete the route simply results in sagging on to the rope. Clearly this should be a less stressful style of climbing and as a result climbers consider top roping to be a preparation activity. $^{6}$

A key aspect of climbing activities is the anxiety that participants often experience and this is acknowledged by writers such as $\mathrm{Hörst}^{7}$ and Godard and Neumann ${ }^{8}$ who include anxiety 2 management as a fundamental skill for the accomplished rock climber. The tendency of climbing to induce anxiety means that measuring an objective stress marker could provide a useful link in understanding the relationship between the 
subjective experience of rock climbing and the situation itself.

Cortisol levels have often been used as an objective marker for stress. $^{9}$ Acute stressors including examinations and exhaustive exercise have been shown to result in an increase in cortisol levels. ${ }^{10}$ However, the relationship between cortisol level and stress has not always been clear. Cortisol reactivity in response to stress has been shown to differ between individuals. The reasons suggested for this are varied but Smyth et al ${ }^{11}$ reported that approximately $25 \%$ of their participants showed little or no reactivity. Oswald et al ${ }^{12}$ also suggested that personality impacts on the degree of cortisol response to stress. Men who scored low for extroversion displayed blunted cortisol responses to the stress of a speech and mental arithmetic test.

Pollard ${ }^{9}$ reviewed studies using cortisol as a stress marker and suggested that, although laboratory studies of acute stress have shown increases in cortisol concentrations, in fact there was evidence that strong emotional arousal of any type may increase cortisol levels. She criticised the research paradigm of examining cortisol responses to activities that were a priori assumed to elicit a negative emotional response. Brown et al ${ }^{13}$ indicated that strong positive emotions may also elicit an increase in cortisol levels. Pollard's suggestion was that increased cortisol levels may be more indicative of heightened emotional arousal rather than stress or anxiety levels alone. Even when experimenters have used protocols to examine responses to both positive and negative mood states ${ }^{14}$ there has still been a deliberate selection of "mood inducer" which has been chosen to elicit a particular directional response. Pollard's view was that further research to clarify the relationship between cortisol, stress and positive emotional arousal would need to present stimuli that could elicit positive or negative emotions related to arousal without the research protocol informing the participants of the emotional response that they were expected to experience.

The current study examined climbers' cortisol and subjective emotional responses to three differing rock climbing conditions. These conditions were designed to provide combinations of higher and lower levels of mental and physical stress. Top roping was employed as a low cognitive, emotional and physical stress condition, lead climbing was employed to provide a high cognitive, emotional and physical stress condition and a third intermediate condition was designed with physical and cognitive demands identical to the leading condition but an emotional demand similar to top roping. This condition had a top rope which provided emotional security and a lead rope that provided a physical and technical task identical to leading. We expected to find higher levels of plasma cortisol concentrations and subjective anxiety rating during more demanding conditions and to be able to relate plasma cortisol concentrations to subjective emotional state. A key aim of this study was to explore how physical and mental stressors can impact on responses to the activity.

\section{METHOD}

Twelve healthy male volunteers (age 20.67 (SD 1.30) years) completed the study. All participants were intermediate rock climbers and had from 3.5 to 4 years' climbing experience; in addition, all had completed a minimum of a 14 week training programme that included lead climbing. The 12 participants were all engaged in climbing activities two to three times per week at the time of the study. The mean height of participants was 1.77 (SD 0.07) m, weight was 69.61 (SD 5.7) kg and body fat 9.0 (SD 3.2)\% . All participants were fully informed of the nature of the study, signed consent statements and were aware that they could withdraw from the study at any stage. Approval for the study was granted by the University Ethical Committee.

\section{Procedure}

Climbers completed an initial familiarisation climb on the route which acted as a vetting process ensuring that volunteers were able to climb at the required level. The familiarisation was to make the three test conditions more comparable by ensuring that climbers had prior experience of the route and the data collection methods before all test conditions. Participants were assigned a climbing schedule for the three test conditions. Climbers completed the route in "lead" condition, "top-rope" condition and "lead and top-rope" condition in a randomised order.

The lead condition replicated the generally accepted best way to ascend a rock climbing route ${ }^{5}$ and the climber was required to work out and execute the moves required to link the holds and manage the safety rope during the ascent. The maximum fall on the set route would have been no more than $2 \mathrm{~m}$. The top-rope condition replicated a training practice whereby the climb is ascended with the safety rope already in place. The lead and top-rope condition was designed to maintain the physical and decision-making task demands of managing the safety rope during climbing whilst eliminating the prospect of actually falling, thereby reducing the emotional control demand. In this condition climbers trailed a rope that they clipped into the onroute anchors but were also secured by the top rope and so would sag onto the top rope rather than fall onto the lead rope if they were to fail on the climb. This condition is unusual for experienced rock climbers but allowed a unique insight into the contribution of the emotional control element to the full task demands of lead climbing. We expected to see an intermediate level of stress for this condition, with leading producing the highest level of stress and top roping the lowest.

All climbers were asked to refrain from exercise on the day prior to testing and to avoid eating within $2 \mathrm{~h}$ of testing. A minimum of 2 days was set between trials. Trials for each climber were set at the same time of day to control for diurnal rhythm variations in cortisol. Climbers also completed the same warm-up prior to each trial, which included 5 minutes of light jogging, sport-specific mobilisation exercises and a climbingspecific warm-up on the climbing wall.

\section{Subjective anxiety assessment}

Anxiety and self confidence were assessed using a Competitive State Anxiety Inventory (CSAI-2R) questionnaire ${ }^{15}$ completed by participants in order to provide a subjective assessment of anxiety levels during climbing. This CSAI-2R was completed immediately after climbing and participants were asked to summarise what they had felt on the route. Although not strictly a competitive event, the route was set at a level where the participants found it very challenging and the climbers were asked to respond to questions relating to competition with completion of the route in mind.

\section{Plasma cortisol concentrations}

Plasma cortisol concentrations were calculated from capillary blood samples. Blood was taken from the little finger of the non-preferred hand in order to have as little impact on climbing as possible. Blood samples $(300 \mu \mathrm{l})$ were collected using lithiumheparin CB300LH Microvettes (Sarstedt Aktiengesellschaft \& Co., Nümbrecht, Germany). Blood samples were kept on ice before being spun for 5 minutes at $4^{\circ} \mathrm{C}$ and $10000 \mathrm{rpm}$ in a 
330R centrifuge (Centurion Scientific, Ford, UK) within 15 minutes of collection. The plasma from each sample was pipetted into a $0.5 \mathrm{ml}$ Eppendorf microtube (Sarstedt Aktiengesellschaft \& Co., Nümbrecht, Germany) and kept at $-20^{\circ} \mathrm{C}$ until analysis. Three $20 \mu \mathrm{l}$ plasma samples were analysed for each trial and variation between samples was no greater than $5.85 \%$. Plasma cortisol concentrations were assayed using a RE52061 Cortisol ELISA Kit (Immuno Biological Laboratories, Hamburg, Germany) and read using a Tecan GENios XFLUOR4 Version: V 4.30 microplate reader (Tecan, Grödig, Austria) at a wavelength of $450 \mathrm{~nm}$. Sigmaplot version 10 (SYSTAT, London, UK) was used to plot absorbances against standard readings for each assay plate to obtain cortisol concentrations.

\section{Statistical analysis}

Differences between state anxiety levels in the three conditions were assessed using separate one way repeated measures analyses of variance (RM ANOVA) for cognitive anxiety, somatic anxiety and self-confidence. Plasma cortisol concentration response was assessed by RM ANOVA for the three climbing conditions. Plasma cortisol concentration delta $(\Delta)$ scores were used in order to account for the variations in initial plasma cortisol concentrations resulting from interindividual and intraindividual differences. Post hoc planned comparisons using polynomial contrasts were undertaken where appropriate. Regression analyses were calculated to explore the extent to which plasma cortisol concentrations could be predicted from anxiety scores. As participants were used more than once Scott and Holt's ${ }^{16}$ inflation factor was applied in order to correct for non-independence of the sample.

\section{RESULTS}

RM ANOVA showed significant differences for somatic anxiety $\left(F_{2,22}=7.74, \quad p=0.009, \quad \eta^{2}=0.41\right) \quad$ and self-confidence $\left(F_{2,22}=9.52, \mathrm{p}=0.001, \eta^{2}=0.46\right)$ scores but not for cognitive anxiety. Subsequent polynomial comparisons showed a linear contrast for both somatic anxiety $\left(F_{1,11}=9.28, \mathrm{p}=0.011\right.$, $\left.\eta^{2}=0.46\right)$ and self-confidence $\left(F_{1,11}=13.98, \quad p=0.003\right.$, $\left.\eta^{2}=0.56\right)$. As can be seen in fig 1 , somatic anxiety was highest in the leading condition and lowest in the top rope condition. Self-confidence, however, was highest in the top rope condition and lowest in the lead condition. Whilst the absolute scores for cognitive anxiety were greatest in the high stress condition and lowest in the low stress condition they were not statistically different.

A RM ANOVA examining $\Delta$ plasma cortisol concentration showed a significant difference $\left(F_{2,22}=3.71, \mathrm{p}=0.041\right.$, $\left.\eta^{2}=0.25\right)$. Cortisol concentrations in the highest stress situation rose whilst cortisol concentrations in the least stressful situation actually dropped during the activity. Polynomial comparisons showed a linear contrast $\left(F_{1,11}=5.07, \mathrm{p}=0.046\right.$, $\left.\eta^{2}=0.32\right)$.

\section{Regression analyses}

Regression analysis showed a cubic relationship between cognitive anxiety and cortisol $\left(R^{2}=0.425, p<0.001\right)$. This relationship can be seen in fig 3 . For very low cognitive anxiety scores, between 10 and 15 points on the CSAI-2R scale, cortisol decreased as anxiety increased. Above cognitive anxiety scores of approximately 16 there was a steady increase of plasma cortisol concentration as cognitive anxiety score increased.

The relationship between self-confidence and plasma cortisol concentration (fig 4) was also cubic $\left(R^{2}=0.281, p=0.004\right)$. The curve for self-confidence and plasma cortisol concentration shows the lowest plasma cortisol concentrations corresponding to a CSAI-2R self-confidence score of approximately 31 .

Regression analysis also showed a cubic relationship between somatic anxiety and cortisol $\left(R^{2}=0.268, p=0.017\right)$. Generally higher levels of reported somatic anxiety were associated with higher levels of plasma cortisol concentrations (fig 5); however, an initial rise in cortisol concentrations was followed by a plateau between approximately 15 and 21 on the somatic anxiety scale (CSAI -2R), after which the rising cortisol levels continued.

\section{DISCUSSION}

Subjective somatic anxiety scores and self-confidence were significantly different in the three conditions. Trends were as expected. The most stressful condition resulted in the highest somatic anxiety rating and lowest self-confidence rating. Polynomial analyses showed that these were linear contrasts with the intermediate condition (lead and top rope) lying between the two more extreme conditions. The increased emotional element of the full lead condition resulted in significantly different responses from the lead and top rope condition. This is an important finding, as physiological studies have tended to operate from a position that assumes physical load is the key component in climbing responses.

The trend for cognitive anxiety was similar. The most stressful condition produced the most cognitive anxiety and the least stressful condition produced the least cognitive anxiety; however, no significant differences were found for cognitive anxiety. This finding was unexpected. Intuitively one might expect increasingly stressful conditions to produce significantly increasing levels of cognitive anxiety.

These results indicate that physical and psychological load need to be considered in any study of rock climbing responses or performance. We would suggest that there is a different impact on anxiety and confidence levels when participants are required to manage their own safety rope, and when falling has more significant consequences.

Cortisol responses $(\Delta)$ were different for each climbing condition with a small increase in plasma cortisol concentration for the most stressful condition (lead), no change for lead and top rope and a reduction in cortisol for the least stressful condition (top rope). RM ANOVA with preplanned polynomial contrasts showed that this was a linear effect.

Regression analyses showed cubic relationships between selfconfidence, somatic and cognitive anxiety, and plasma cortisol concentration. Figure 5 shows that increases in cortisol concentration were generally associated with increases in reported somatic anxiety symptoms, except for a plateau in cortisol concentration which occurred around $90 \mathrm{ng} / \mathrm{ml}$ and corresponded to somatic anxiety scores between 15 and 21 points. The positive trend would support Pollard's ${ }^{9}$ view that arousal can result in increases in cortisol levels. However, in this study cortisol changes were most noticeable when arousal symptoms (somatic anxiety) were very weak or moderately to very strong. A moderate degree of somatic anxiety led to quite undifferentiated cortisol responses.

The best fit line for cognitive anxiety and plasma cortisol concentration (fig 2) is particularly interesting as the relationship it describes is cubic. The highest cortisol levels were still found when cognitive anxiety was high, but the lowest levels of cortisol were actually found when cognitive anxiety scores were approximately 16 points. A possible explanation of this phenomenon might be that between 10 and 15 points cognitive 


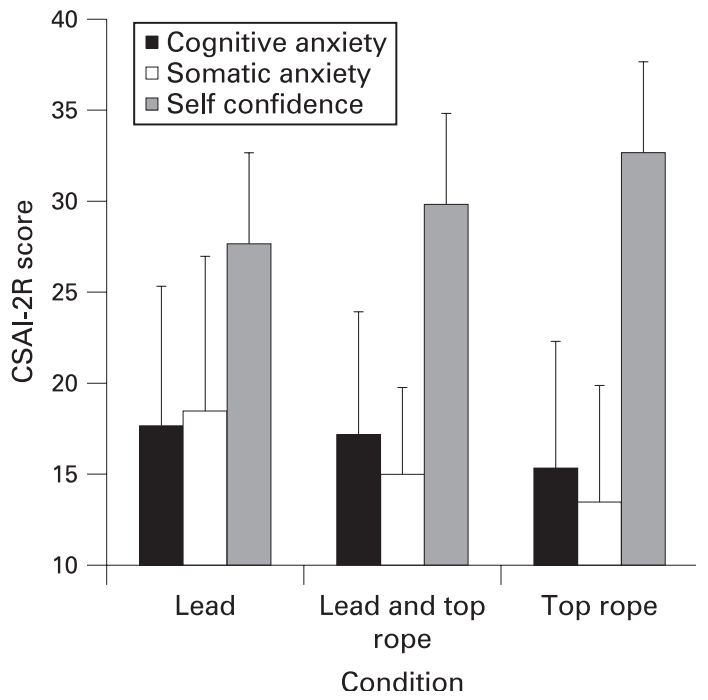

Figure 1 Subjective anxiety and self-confidence rating for each climbing condition

anxiety is not the most dominant emotion and that arousal levels may actually be driven by an alternate emotion. The selfconfidence scale of the CSAI-2R is normally found to correlate negatively with the cognitive anxiety scale, and so is sometimes considered unnecessary by researchers. ${ }^{17}$ In this case it may indicate positive emotional elements becoming more dominant as cognitive anxiety drops to very low levels. Further research could investigate this possibility.

An important aspect of the present study was that mood states were induced naturally by an activity that could, and did, evoke a spectrum of anxiety and self confidence responses. Brown et $a l,{ }^{13}$ who found that both elation and sadness resulted in increases of serum cortisol concentration, have expressed concern that too often studies have been set up in such a way that participants were given unambiguous expectations of the mood changes that experimenters wanted to study, and have pointed out that this has been shown to lead to the expected moods being reported. Meta-analysis showed that participants have often reported more extreme mood changes when experimental design made clear the intentions of experimenters to manipulate moods.

In conclusion, the present study indicates that research into cortisol responses to naturally occurring, positive and negative, mood states during controlled stressors could lead to a fuller understanding of cortisol responses. Activities which have the potential to elicit strong mood states in either direction may provide an experimental platform to support this kind of research.

Competing interests: None.

\section{REFERENCES}

1. Watts PB, Martin DT, Durtschi S. Anthropometric profiles of elite male and female competitive sport rock climbers. J Sports Sci 1993:11:113-17.

2. Draper N, Bird EL, Coleman I, et al. Effects of active recovery on lactate concentration, heart rate and RPE in climbing. J Sports Sci Med 2006;5:97-105.

3. Watts $\mathbf{P}$, Newbury V, Sulentic J. Acute changes in handgrip strength, endurance and blood lactate with sustained sport rock climbing. J Sports Med Phys Fitness 1996;36:255-60.

4. Williams ES, Taggart P, Carruthers M. Rock climbing: Observations on heart rate and plasma catecholamine concentrations and the influence of oxprenolol. Br J Sports Med 1978:12:125-8.

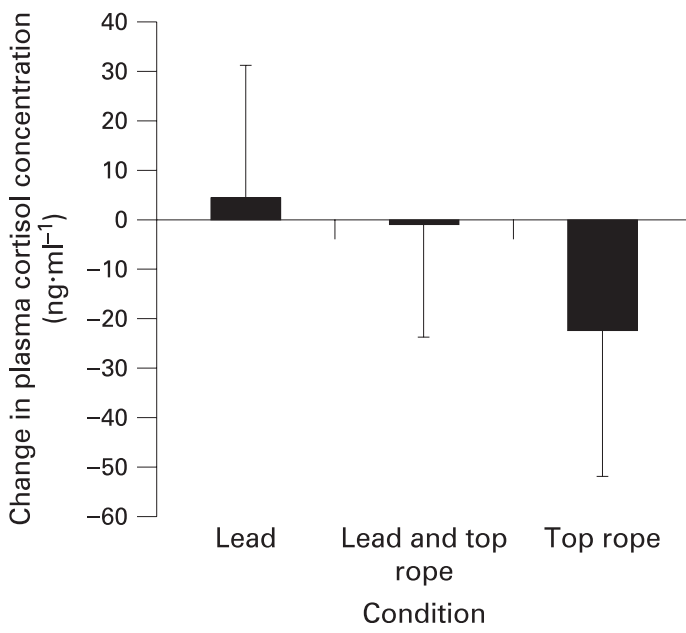

Figure 2 Change in plasma cortisol concentration for each condition

5. Tejada-Flores L. Games climbers play. In: Wilson K, ed. The games climbers play London: Bâton Wicks, 2000.

6. Richardson A. Rock climbing for instructors. Wiltshire: The Crowood press, 2001

7. Hörst EJ. Training for climbing. Connecticut: Falcon Guide, 2003

8. Goddard D, Neumann U. Performance rock climbing. PA: Stackpole Books, 1993.

9. Pollard TM. Use of cortisol as a stress marker: practical and theoretical problems. Am J Human Biol 1995; 7:265-74.

10. Hellhamer DH, Heib C, Hubert W, et al. Relationships between salivary cortisol release and behavioural coping under examination stress. IRCS Med Sci 1985:13:1179-80

11. Smyth J, Ockenfels MC, Porter $L$, et al. Stressors and mood measured on a momentary basis are associated with salivary cortisol secretion. Psychoneuroendocrinology 1998;23:353-70.

12. Oswald LM, Zandi P, Nestadt G, et al. Relationship between cortisol responses to stress and personality. Neuropsychopharmacology 2006;31:1583-91.

13. Brown WA, Sirota AD, Niaura R, et al. Endocrine correlates of sadness and elation Psychosom Med 1993;55:458-67.

14. Hubert W, de Jong-Meyer R. Salivary cortisol responses to sad and humorous film segments. In: Kirschbaum C, Read GF, Hellhammer DH, eds. Assessment of hormones and drugs in saliva in biobehavioural research. Seattle: Hogrefe \& Huber Publishers, 1992.

15. Cox RH, Martens MP, Russell WD. Measuring anxiety in athletics: The revised competitive state anxiety inventory-2. J Sport Exercise Psychol 2003:25:519-33.

16. Scott AJ, Holt D. The effect of two-stage sampling on ordinary least squares methods. J Am Stat Assoc 1982;77:848-54.

17. Gill DL. Psychological dynamics of sport and exercise. Champaign, Illinois: Human Kinetics, 2000.

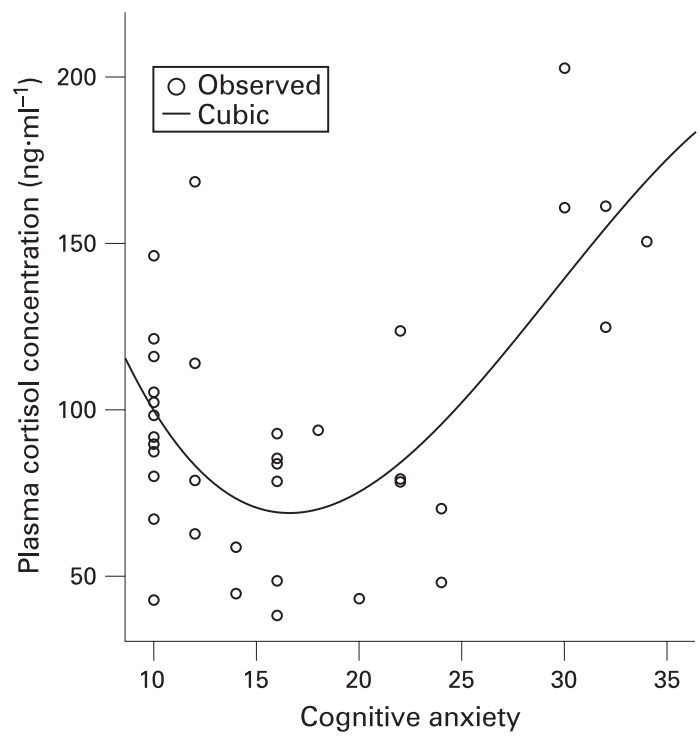

Figure 3 The relationship between plasma cortisol concentration and CSAI-2R cognitive anxiety score 


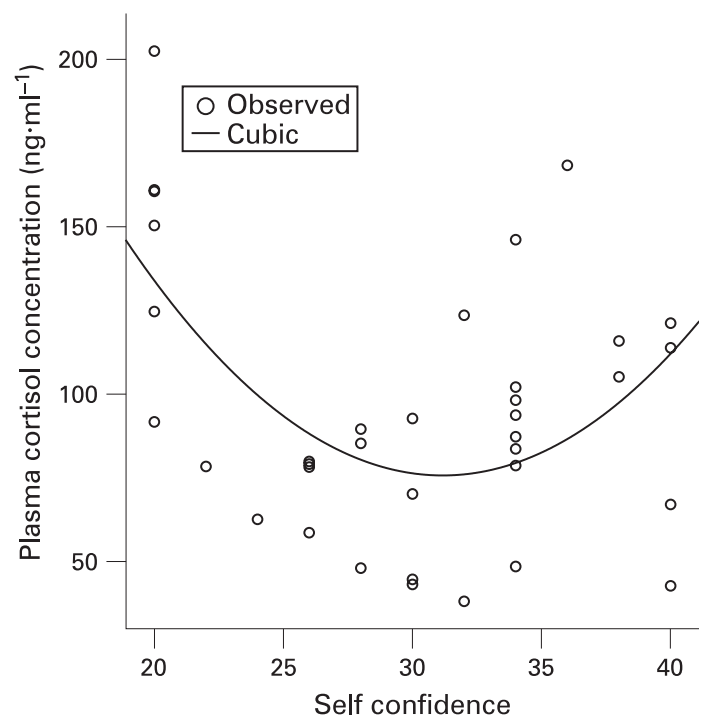

Figure 4 The relationship between plasma cortisol concentration and CSAI-2R self-confidence score

Authors Queries

Journal: British Journal of Sports Medicine

Paper: sm46011

Title: Perceived anxiety and plasma cortisol concentrations following rock climbing with differing safety rope protocols

Dear Author

During the preparation of your manuscript for publication, the questions listed below have arisen. Please attend to these matters and return this form with your proof. Many thanks for your assistance

\begin{tabular}{|l|l|l|}
\hline $\begin{array}{l}\text { Query } \\
\text { Reference }\end{array}$ & Query & Remarks \\
\hline 1 & $\begin{array}{l}\text { You have opted not to pay a fee to } \\
\text { make your article free online } \\
\text { (Unlocked). If you wish to change } \\
\text { your mind, please indicate this } \\
\text { clearly and provide invoice } \\
\text { address and contact details when } \\
\text { you return your proofs. This is the } \\
\text { last point in the production process } \\
\text { where you can choose to Unlock } \\
\text { your paper; the cost of this service } \\
\text { is £1,200/\$2,220/€1,775 (+VAT) } \\
\text { and further details can be found at } \\
\text { http://bjsm.bmj.com/info/unlocked. } \\
\text { dtl }\end{array}$ & \\
\hline 2 & $\begin{array}{l}\text { Goddard (ref. 8) is cited as Gc. } \\
- \text { please confirm which is corr What is already known on this topic }\end{array}$ & \\
\hline
\end{tabular}

There is very little research on the psychological impact of rock climbing, and none links subject anxiety ratings to plasma cortisol concentrations. Studies have examined the effects of acute stress on cortisol, but these have tended to be in situations with very clear positive or negative emotional connotations. Stressors that have more open-ended interpretations have received little attention in psychobiological research. 


\section{Original article}

\section{What this study adds}

These results show that there is a link between the task demands of differing rock climbing protocols and the psychobiological status of rock climbers. Increasing task demands impact on both subjective anxiety responses and plasma cortisol concentrations. In this study rock climbing provides a stressor which can be interpreted in a more open-ended manner by participants than many research protocols have allowed, and this means that subjective responses are relatively free from suggestibility effects. Changes in plasma cortisol concentrations relate to task demand and are linked to cognitive anxiety, somatic anxiety and self confidence ratings. 